\title{
Cryopreserved biopsy tissues of rectal cancer liver metastasis for assessment of anticancer drug response in vitro and in vivo
}

\author{
YUAN ZHANG ${ }^{1,2^{*}}$, WEI-JIAN HUANG ${ }^{3 *}$, QIU-RUI YANG ${ }^{4}$, HONG-DAN ZHANG $^{4}$, XUE-JING ZHU ${ }^{4}$, \\ MIN ZENG $^{3}$, XU ZHOU ${ }^{3}$, ZHEN-YU WANG ${ }^{1}$, WEI-JIAN LI ${ }^{1}$, HONG-SHU JING $^{1}$, XUE-BIN ZHANG ${ }^{1}$, \\ YAO-PING SHI ${ }^{1}, \mathrm{HAO} \mathrm{HU}^{1}, \mathrm{HE}-\mathrm{XIN} \mathrm{YAN}^{1,3}, \mathrm{ZONG}^{-\mathrm{HAI} \mathrm{LI}^{2}}$ and BO ZHAI ${ }^{1}$ \\ ${ }^{1}$ Department of Tumor Interventional Oncology, Renji Hospital, School of Medicine, Shanghai Jiaotong University, \\ Shanghai 200127; ${ }^{2}$ State Key Laboratory of Oncogenes and Related Genes, Shanghai Cancer Institute, Renji Hospital, \\ School of Medicine, Shanghai Jiaotong University, Shanghai 200032; ${ }^{3}$ International Cooperation Laboratory \\ on Signal Transduction, Eastern Hepatobiliary Surgery Institute, The Second Military Medical University, \\ Shanghai 200438; ${ }^{4}$ Shanghai Celliver Biotechnology Co., Shanghai 201203, P.R. China
}

Received January 17, 2018; Accepted June 14, 2019

DOI: $10.3892 /$ or.2019.7450

\begin{abstract}
Living tumors are of great scientific value for clinical medicine and basic research, especially for drug testing. An increasing number of drug tests fail due to the use of imperfect models. The aim of the present study was to develop a novel method combining vitrification-based cryopreservation of tumor biopsies and precision-cut slice cultivation for the assessment of anticancer drug responses. Biological characteristics of rectal cancer liver metastasis biopsies could be retained by vitrification-based cryopreservation. The patient-derived xenograft models were successfully established using both fresh and warmed biopsy tissues. Precision-cut slicing provided a similar three-dimensional architecture and heterogeneity to the original tumor. The positive drug responses in the xenograft model were consistent with those in precision-cut slice cultures in vitro. The present
\end{abstract}

Correspondence to: Dr Bo Zhai, Department of Tumor Interventional Oncology, Renji Hospital, School of Medicine, Shanghai Jiaotong University, 160 Pujian Road, Shanghai 200127, P.R. China

E-mail: zhaiboshi@sina.com

Professor Zong-Hai Li, State Key Laboratory of Oncogenes and Related Genes, Shanghai Cancer Institute, Renji Hospital, School of Medicine, Shanghai Jiaotong University, 25/Ln. 2200 Xie Tu Road, Shanghai 200032, P.R. China

E-mail: zonghaili@shsmu.edu.cn

*Contributed equally

Abbreviations: RLM, rectal cancer liver metastasis; PDX, patient-derived xenograft; H\&E, hematoxylin and eosin; IHC, immunohistochemistry; OXA, oxaliplatin; KEGG, Kyoto Encyclopedia of Genes and Genomes

Key words: biopsy, vitrification-based cryopreservation, precision-cut slice, RLM, OXA study demonstrated that live tumor biopsies could be preserved using vitrification-based cryopreservation. The warmed tissues developed xenograft tumors, which were also useful for either in vivo or in vitro anticancer drug testing. Precision-cut slices derived from the warmed tissues provided an efficient tool to assess anticancer drug response in vitro.

\section{Introduction}

Colorectal cancer, including rectal cancer, is the third most prevalent malignancy worldwide and a leading cause of cancer-related deaths in the developed world (1). Synchronous distant metastases are diagnosed in approximately $15-20 \%$ of rectal cancer patients, most commonly involving the liver (2). Surgery remains the standard of care for rectal cancer liver metastasis (RLM). However, $85 \%$ of patients with RLM are considered unresectable (3). Those patients require systemic chemotherapy, in the form of a fluoropyrimidine doublet (FOLFOX/CAPOX or FOLFIRI/CAPIRI) combined with a biologic targeting either angiogenesis, or epidermal growth factor receptor in patients with RAS wild-type tumors (4). Unfortunately, many patients do not respond to first-line chemotherapy, or develop resistance. Consequently, further cancer research in drug testing and optimized personalization strategies for RLM treatment are urgently needed.

In previous years, established cell lines have been widely used in many aspects of medical research, and particularly as in vitro models in preclinical drug testing (5). However, these are an imperfect model because the three-dimensional architecture of the tumor tissue and cell-cell communications that clearly exist in vivo are lost, and therefore they do not faithfully reflect the tumor of origin (6). Recently, various novel strategies have been applied to maintain or reconstitute an environment closely resembling the tumor tissue, such as two-dimensional culture of dissociated tumor cells and three-dimensional spheroid cultures (7). However, these still cannot imitate the intricate tissue architecture and the high degree of variability seen in individual tumors. It is 
becoming increasingly clear that the progression of cancer and the response to chemotherapeutic drugs mainly depends on specific intercommunications between tumor cells and surrounding tissue components (8). Therefore, an ideal model is required for the accurate characterization of chemotherapy sensitivity in RLM tumors from patients.

A potentially desirable model was provided by precision-cut slicing, which maintains the complete tissue architecture and full heterogeneity of the tumor in vivo $(9,10)$. However, there is no standard approach to cultivating tumor slices in vitro. Besides, slice cultivation derived from fresh biopsy tissue, which is convenient to obtain and preserve, is rarely reported. Another problem is that no preservation method has thus far been applied to maintain living fresh tissue, to the best of our knowledge. Currently, the conventional preservation techniques for fresh tumor tissue, either as formalin-fixed paraffin embedded samples or by flash freezing in liquid nitrogen, lead to the absolute inactivation of the fresh tissue and can only provide morphological and genetic information, which has led to the infrequent utilization of fresh specimens. Recently, a standardized vitrification-based cryopreservation method has been developed to preserve fresh tumor tissue, by which the biological characteristics of the original tumor can be retained and the utilization of specimens may be markedly improved.

The present study combined the vitrification-based cryopreservation method with the precision-cut slicing technique, using available tumor tissues from a mouse model of human RLM biopsy to assess anticancer drug responses.

\section{Materials and methods}

Collection of human RLM biopsy tissues. Fresh sterile biopsy tissues were obtained from patients with RLM at the Department of Interventional Oncology, Renji Hospital Affiliated to Shanghai Jiaotong University School of Medicine (Shanghai, China). Samples were collected between June 2016 and December 2016. There were a total of 20 patients, including 10 men and 10 women, with a mean age of 60 years. The pathological diagnosis of all patients was RLM and none had received any prior treatment. The fresh biopsy tissues were then directly transported to the laboratory within $2 \mathrm{~h}$. All specimens were kept at $4^{\circ} \mathrm{C}$ on ice and transported in preservation medium (Tissue Mate ${ }^{\mathrm{TM}}$; Celliver Biotechnology, Inc.) which is composed of energy substrate, iron chemicals and antioxidants, without protein. This investigation was approved by the ethics committee of Renji Hospital and all patients provided written informed consent. Details are illustrated in Fig. 1.

Cryopreservation and warming procedures. All cryopreservation solutions (cat. no. LT2601; LiveTissue ${ }^{\mathrm{TM}}$ ) and warming solutions (cat. no. LT2602; LiveTissue ${ }^{\mathrm{TM}}$ ) were provided by Celliver Biotechnology, Inc. For tissue cryopreservation, vitrification solution 1 (V1), vitrification solution 2 (V2) and vitrification solution 3 (V3) were pre-warmed in a $26^{\circ} \mathrm{C}$ water bath. Fresh RLM biopsy tissues were cleaned twice with sterile PBS and transferred into $5 \mathrm{ml} \mathrm{V} 1,5 \mathrm{ml} \mathrm{V} 2$ and $5 \mathrm{ml} \mathrm{V} 3$ for 3, 3 and $6 \mathrm{~min}$, respectively. Tissues were then placed onto a thin metal strip and submerged into liquid nitrogen for at least 5 min. Finally, the strips with tissues were placed into frozen storage tubes and preserved in the nitrogen canister. The tissue samples were stored in the liquid nitrogen until warming. For tissue warming, the frozen storage tubes were removed from the nitrogen canister and the strips with the cryopreserved biopsy tissues were quickly transferred into $10 \mathrm{ml}$ warming solution 1 , and incubated for $3 \mathrm{~min}$ in a $37^{\circ} \mathrm{C}$ water bath. The tissues were then transferred into $5 \mathrm{ml}$ warming solution 2 and $5 \mathrm{ml}$ warming solution 3 for 5 and $10 \mathrm{~min}$, respectively, at $26^{\circ} \mathrm{C}$. Warmed tissues were cleaned twice with sterile PBS and kept on ice until use.

Tissue slice preparation and cultivation. Warmed tissues were used to establish the first generation of patient-derived xenografts (PDXs), in order to produce ample tumor tissues for further experiments. Subcutaneous xenografts were cut into $300-\mu \mathrm{M}$-thick precision-cut slices using a microtome for slice preparation [slices of $300 \mu \mathrm{M}$ were considered to be the most suitable thickness for RLM after conducting several slicing experiments with different thicknesses (data not shown)].

For precision-cut slice preparation, tissues were embedded in $2 \%$ low temperature gelling agarose (Sigma-Aldrich; Merck $\mathrm{KGaA}$ ) and $300-\mu \mathrm{m}$-thick slices were prepared using a VF-300 Microtome (Bio-Gene Technology, Ltd.). Parameter settings, such as the frequency and amplitude of vibration slicing, were determined by the specific tumor texture. Tissue slices (diameter, $2 \mathrm{~mm}$ ) were then prepared using a hand-held coring tool, and all the procedures were performed under sterile conditions.

Precision-cut slices were maintained on Transwell inserts (pore size, $0.4 \mu \mathrm{m}$; Corning, Inc.), with two slices to each insert. Cultivation was performed in 24-well plates containing $450 \mu 1$ RPMI-1640 medium (BasalMedia) with 10\% fetal bovine serum (Biological Industries), penicillin (100 U/ml; BasalMedia) and streptomycin (100 U/ml; BasalMedia), and kept at $37^{\circ} \mathrm{C}$ in a humidified incubator with $5 \% \mathrm{CO}_{2}$. For drug testing of slices in vitro, oxaliplatin (OXA; MedChemExpress, LLC) was used and tested at a concentration of $20 \mu \mathrm{M}$. Drug testing commenced after $24 \mathrm{~h}$ of slice culture and was performed for an additional $72 \mathrm{~h}$. Medium changes were performed every $24 \mathrm{~h}$.

Experimental methods for $m R N A$ sequencing. RNA purity was checked using the kaiaoK5500 ${ }^{\circledR}$ Spectrophotometer (Beijing Kaiao Technology Development Co., Ltd). RNA integrity and concentration was assessed using the RNA Nano 6000 Assay kit and the Bioanalyzer 2100 system (Agilent Technologies, Inc.). A total amount of $2 \mu \mathrm{g}$ RNA/sample was used as input material for the RNA sample preparations. Sequencing libraries were generated using NEBNext ${ }^{\circledR}$ Ultra $^{\text {TM }}$ RNA Library Prep kit for Illumina ${ }^{\circledR}$ (cat. no. E7530L; New England BioLabs, Inc.), following the manufacturer's recommendations, and index codes were added to attribute sequences to each sample. Briefly, mRNA was purified from the total RNA using poly-T oligo-attached magnetic beads. Fragmentation was carried out using divalent cations under elevated temperature in NEBNext First Strand Synthesis Reaction Buffer (5X). First strand cDNA was synthesized using random hexamer primer and RNase H. Second strand cDNA synthesis was subsequently performed using buffer, dNTPs, DNA polymerase I and RNase H. The library fragments were purified 
with QiaQuick PCR kits (Qiagen, Inc.) and elution with EB buffer, then terminal repair, A-tailing and adapter adding were implemented. The products were retrieved and PCR was performed, then the library was completed. The RNA concentration of the library was measured using a Qubit ${ }^{\circledR}$ RNA Assay kit in Qubit $^{\circledR} 3.0$ (Thermo Fisher Scientific, Inc.)for preliminary quantification, and then diluted to $1 \mathrm{ng} / \mu 1$. Insert size was assessed using the Agilent Bioanalyzer 2100 system (Agilent Technologies, Inc.), and qualified insert size was accurately quantified using the StepOnePlus ${ }^{\mathrm{TM}}$ Real-Time PCR System (Thermo Fisher Scientific, Inc.; library valid concentration, $>10 \mathrm{nM}$ ). The clustering of the index-coded samples was performed on a cBot cluster generation system using a HiSeq PE Cluster kit v4-cBot-HS (Illumina, Inc.) according to the manufacturer's instructions. After cluster generation, the libraries were sequenced on an Illumina, Inc. platform and $150 \mathrm{bp}$ paired-end reads were generated. The variations in gene expression could be detected by different colors in the heat map. Kyoto Encyclopedia of Genes and Genomes (KEGG) enrichment exhibited the main types and functions of the detected differential genes. Kyoto Encyclopedia of Genes and Genomes (KEGG) pathway analysis was processed using KOBAS 3.0 (http://kobas.cbi.pku.edu.cn/).

Establishment of xenograft model. A total of 20 female NOD/SCID mice of 6-8 weeks old were housed and treated under specific pathogen-free conditions at the Experimental Animal Center of Shanghai Jiaotong University School of Medicine. Mice were housed in a 12-h light/dark cycle with food and water available at all times. The room temperature was maintained at $24 \pm 1^{\circ} \mathrm{C}$ and relative humidity at $50 \%$. The average weight of the animals was $18 \mathrm{~g}$. All mice were purchased from the Shanghai Experimental Center of Chinese Academy of Science. All the experimental procedures performed on the animals were approved by the Shanghai Medical Experimental Animal Care Commission.

Mice were implanted subcutaneously with warmed biopsy tissues on the right flank, and tumor growth was monitored twice a week. When the tumor burden was $\sim 500 \mathrm{~mm}^{3}$ [tumor volume was measured with calipers and calculated using the formula $\mathrm{V}=1 / 2$ (length $\mathrm{x}$ width ${ }^{2}$ )], the mice were sacrificed and the tumors were used for continuous passaging to the third generation. The subcutaneous xenograft tumors of PDXs derived from warmed biopsy tissues were cut into 1-mm-thick slices in a metal mold before cryopreservation. When the tumor burden was $100-150 \mathrm{~mm}^{3}$, mice with xenografts derived from fresh tissue and warmed tissue were injected intraperitoneally with OXA in $200 \mu \mathrm{l}$ PBS at a dose of $5 \mathrm{mg} / \mathrm{kg}$ (twice a week for 4 weeks). All the mice in the PBS group were injected intraperitoneally with $200 \mu \mathrm{l}$ PBS. The mice in the control group were injected with nothing. The mice were sacrificed 4 weeks later and the tumors were used for hematoxylin and eosin (H\&E)/immunohistochemical (IHC) staining.

Cell Counting Kit-8 (CCK-8) assay. A CCK-8 assay (Dojindo Molecular Technologies, Inc.) was used to evaluate the viability of tissue slices at each time point $(24,48,72$ and $96 \mathrm{~h})$ during the culturing process in vitro. RPMI-1640 medium (50 $\mu \mathrm{l} /$ well) and CCK-8 solution (10 $\mu \mathrm{l} /$ well) were added into 96 -well plates. The tissue slices were added one slice/well. The plates were kept at $37^{\circ} \mathrm{C}$ in a humidified incubator with $5 \% \mathrm{CO}_{2}$ for $2 \mathrm{~h}$. The slices were removed from the 96-well plates and the plates were transferred to the Thermo Fisher Scientific, Inc. microplate reader (Multiskan GO). The absorbance at $450 \mathrm{~nm}$ was measured and three wells were tested for each sample at each time point. In addition, during preliminary work, the cell viability following different lengths of preservation time in liquid nitrogen were compared by CCK- 8 assay.

Acetoxymethylester of calcein (calcein-AM) cell viabilityassay and Hoechst 33342 staining. The Live/Dead ${ }^{\circledR}$ Viability Assay kit (Nanjing KeyGen Biotech Co., Ltd.) and Hoechst 33342 (Beyotime Institute of Biotechnology) were stored at $-20^{\circ} \mathrm{C}$ and allowed to warm to room temperature prior to experimentation. The viability assay stock reagents (calcein-AM, $4 \mathrm{mM}$ ) were diluted to $1 \mu \mathrm{M}$ in physiological solution and mixed with $2 \mu \mathrm{g} / \mathrm{ml}$ Hoechst 33342 stock reagents at room temperature for $30 \mathrm{~min}$. Representative images were captured with the Leica TCS SP8 confocal microscope (Leica Microsystems GmbH). The ratio of living cells in the calcein-AM cell viability assay/Hoechst 33342 staining was calculated based on manual counting within ten random microscopic fields.

$H \& E / I H C$ staining. Tissue slices were fixed in $10 \%$ phosphate-buffered formalin for at least $24 \mathrm{~h}$ at room temperature and subsequently paraffin-embedded. For the examination of histopathology, paraffin sections $(4 \mu \mathrm{m})$ were stained with $\mathrm{H} \& \mathrm{E}$ at room temperature. Before incubating with antibodies, samples were blocked with $20 \%$ goat serum (cat. no. Ab138478; Abcam) for $15 \mathrm{~min}$ at room temperature. For Ki67 staining, sections were incubated with the primary antibody (cat. no. Ab15580; Abcam; 1:1,000 dilution) and a horseradish peroxidase (HRP)-conjugated secondary antibody (cat. no. Ab205718; Abcam; 1:20,000 dilution). For caspase-3 staining, sections were incubated with the primary antibody (cat. no. Ab184787; Abcam; 1:1,000 dilution) and an HRP-conjugated secondary antibody (cat. no. Ab97051; Abcam; 1:500 dilution). Sections were dewaxed in xylene and rehydrated in an ethanol gradient of 100, 95 and $80 \%$, and heat-mediated antigen retrieval of the tissue sections was carried out at a temperature of $96-98^{\circ} \mathrm{C}$ before they were allowed to cool. Paraffin-embedded sections $(4 \mu \mathrm{m})$ were incubated with primary antibodies overnight at $4^{\circ} \mathrm{C}$. The secondary antibody was used to detect the primary for $1 \mathrm{~h}$ at room temperature. Confocal laser scanning microscopy was performed using an Olympus Corporation BX51 instrument.

Statistical analysis. Statistical evaluations were performed using a Student's t-test and one-way ANOVA with post hoc least significant difference test by IBM SPSS Statistics 22.0 (IBM Corp). $\mathrm{P}<0.05$ was considered to indicate a statistically significant difference. Three repeats were performed.

\section{Results}

Biological characteristics of RLM biopsy tissues are retained by vitrification-based cryopreservation. By conducting several CCK-8 assays during preliminary work, it was found that no difference was induced by different lengths of preservation time in liquid nitrogen (data not shown). All the fresh 


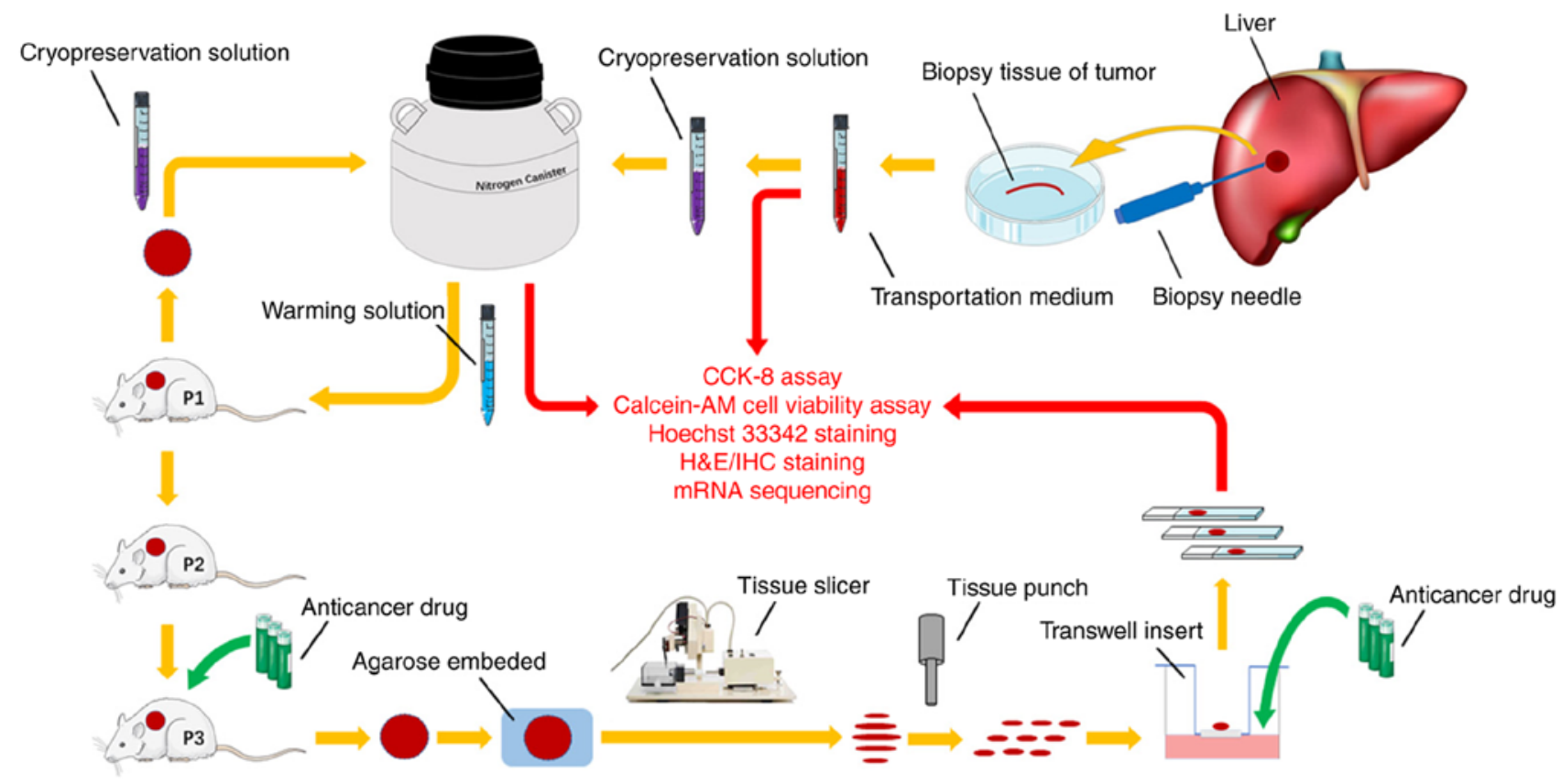

Figure 1. Workflow of cryopreserved slice culture and drug testing. Rectal cancer liver metastasis biopsies were obtained and transported to the laboratory in preservation medium at $4^{\circ} \mathrm{C}$ within $2 \mathrm{~h}$. Tissues were cryopreserved and stored in a nitrogen canister. Warmed tissues were used to establish patient-derived xenografts, which were propagated to further generations. Xenografts were embedded in agarose and cut into 300- $\mu$ m-thick slices using a VF-300 microtome. Tissue slices ( $2 \mathrm{~mm}$ in diameter) were maintained on Transwell inserts and treated with OXA at s concentration of $20 \mu \mathrm{M}$. Mice were injected with OXA in $200 \mu \mathrm{l}$ PBS at a dose of $5 \mathrm{mg} / \mathrm{kg}$. Experiments were performed to evaluate the efficacy of cryopreservation combined with slice cultivation in the assessment of anticancer drug responses by CCK-8 assay and calcein-AM cell viability assay/Hoechst 33342 staining, H\&E/IHC staining and mRNA sequencing. OXA, oxaliplatin; CCK-8, Cell Counting Kit-8; H\&E, hematoxylin and eosin; IHC staining, immunohistochemical staining; P, passage; calcein-AM, acetoxymethyl ester of calcein.

RLM biopsies were obtained from Department of Tumor Interventional Oncology, Renji Hospital, School of Medicine. The study was performed using standard procedures, as depicted in Fig. 1. The freshly collected RLM biopsy tissues were cryopreserved and warmed according to the schedule in Fig. 2A, using cryopreservation solutions (Fig. 2B, left) and warming solutions (Fig. 2B, right). Fresh biopsy tissues (Fig. 2C) were soft and fragile, and required gentle manipulation in the cryopreservation and warming procedures. The cryopreserved tissues (Fig. 2D, top) were found to be translucent and sclerotic, while the warmed tissues (Fig. 2D, bottom) recovered their softness and luster. Xenograft models were successfully established with both fresh biopsy tissues (Fig. 2E, left) and warmed biopsy tissues (Fig. 2E, right). Fresh and warmed tumor biopsies from 20 patients with RLM were implanted into NOD-SCID mice. From these, 11 xenografts derived from 20 fresh RLM biopsies and 10 xenografts derived from 20 warmed RLM biopsies were successfully established (take rates, 55 and 50\%, respectively).

Calcein-AMcell viability assay/Hoechst 33342 staining and $\mathrm{H} \& \mathrm{E} / \mathrm{IHC}$ staining indicated that no obvious difference was detectable between fresh tissues and warmed tissues (Fig. 2F). Tissue viability was assessed by calcein-AM cell viability assay/Hoechst 33342 staining. The living cell ratio was 76.5\% in fresh tissues and $75 \%$ in warmed tissues, which confirmed that cryopreservation had little influence on the biological viability of the tissue (Fig. 2G). H\&E staining showed that the morphological features remained in the warmed tissues, and were similar to those of fresh tissues. The proliferative rate, as assessed by Ki67, was maintained at almost the same level between fresh and warmed tissues, indicating that little damage was induced by the cryopreservation and warming procedures to the tissue proliferative capacity. The CCK- 8 assay illustrated that both warmed and fresh tissues could be cultured in vitro for at least $96 \mathrm{~h}$ (Fig. 2H). From the heat map, it was apparent that the color of left column, representing the warmed tissue, was mostly consistent with that of the fresh tissue on the right. Only a small part of the heat map in the left column was different from the right column, which corresponded to genes with metabolic functions. Therefore, limited variation in gene expression between fresh and warmed tissues was identified by the heat map (Fig. 2I). According to the KEGG enrichment analysis, it was found that many of the differential genes, which were represented by large red dots, were closely related to metabolic processes (Fig. 2J). These results confirmed that the RLM biopsies were applicable to the establishment of PDX models, and that the vitrification-based cryopreservation method was able to largely maintain the biological activity and histological features of the RLM biopsy tissues.

Precision-cut slices provide the complete three-dimensional architecture and full heterogeneity of the original tumor. The subcutaneous xenograft tumors of PDXs derived from warmed biopsy tissues were cut into 1-mm-thick slices in a metal mold before cryopreservation (Fig. 3A). Precision-cut 300- $\mu \mathrm{M}$-thick slices were obtained using a VF-300 microtome (Fig. 3B and C). Tissue slices ( $2 \mathrm{~mm}$ in diameter) were then prepared using a hand-held coring tool and maintained on Transwell inserts (pore size, $0.4 \mu \mathrm{m}$ ) (Fig. 3D).

To test the in vitro anticancer responses of both fresh and warmed slices using OXA, standardized slice culturing 
A
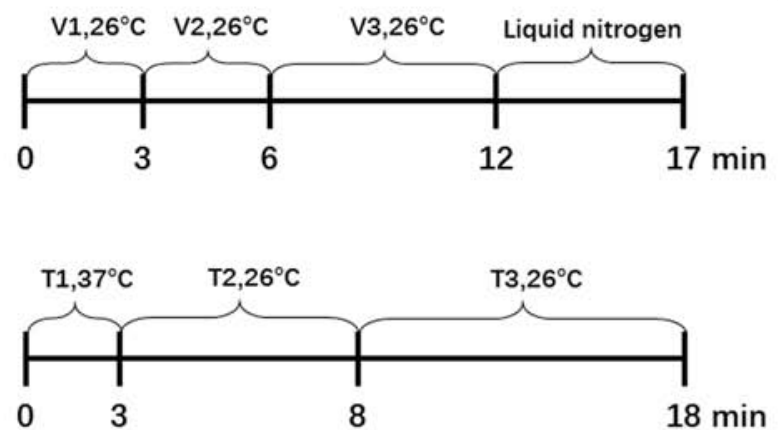

C

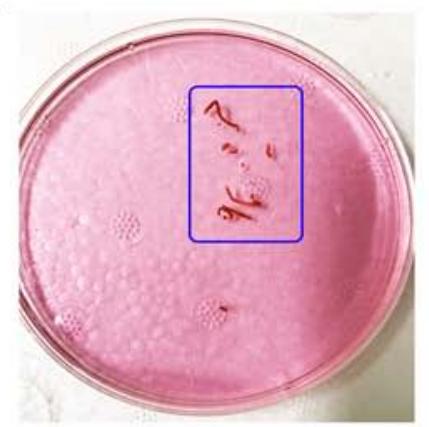

F
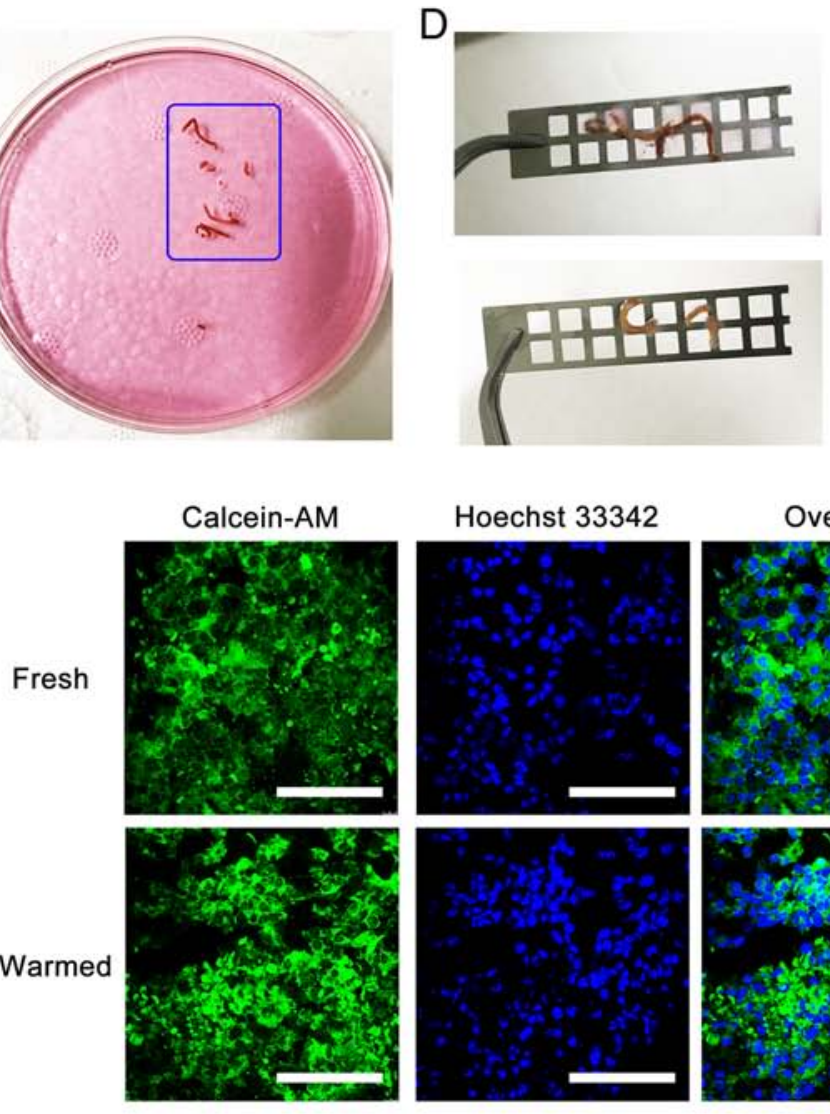

E
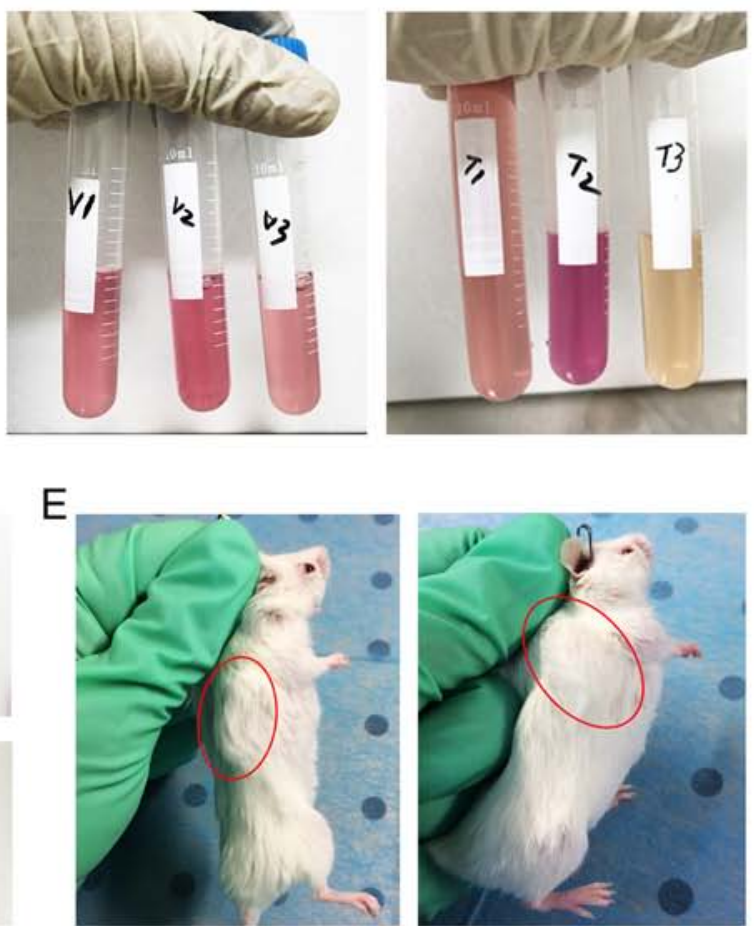

Hoechst 33342
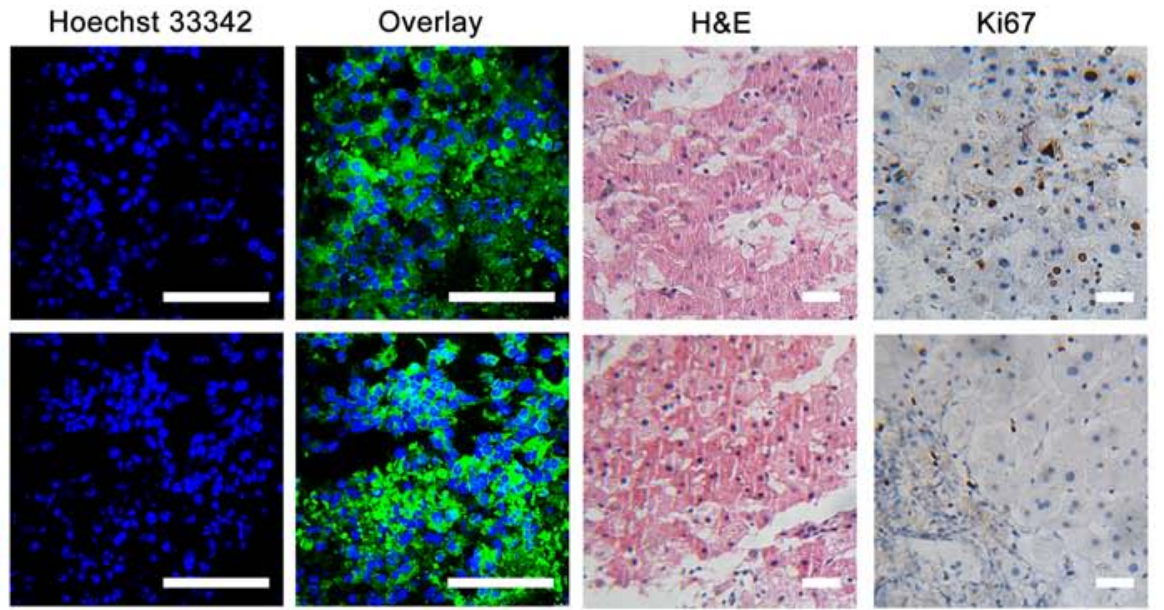

G

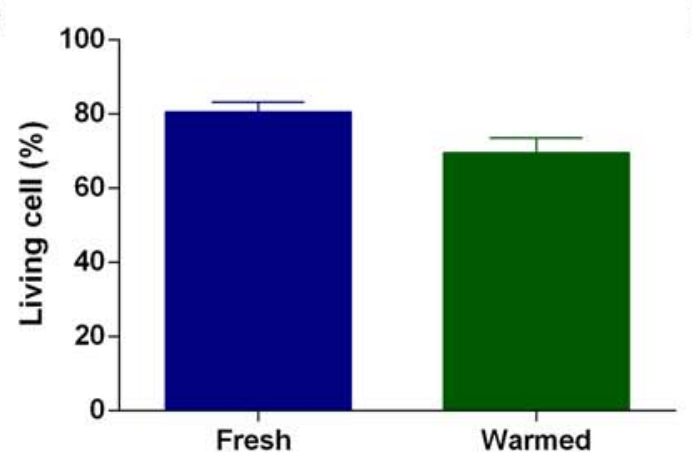

$\mathrm{H}$

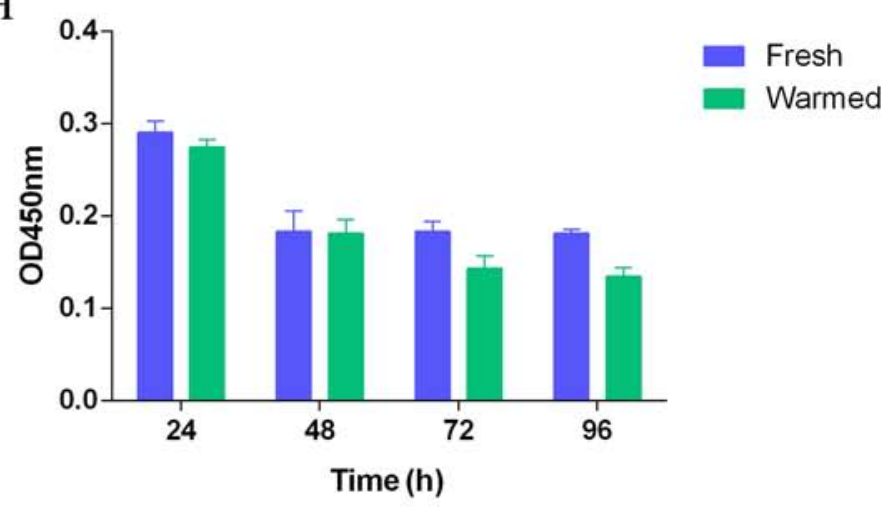

Figure 2. Cryopreservation and warming of RLM biopsy tissue. (A) The time schemes of the cryopreservation (top) and warming procedures (bottom). (B) Cryopreservation solutions (left) and warming solutions (right). (C) Fresh RLM biopsy. (D) Cryopreserved tissues (top) and warmed tissues (bottom). (E) Xenograft model derived from a fresh RLM biopsy tissue (left, $n=11$ ) and warmed RLM biopsy tissue (right, $\mathrm{n}=10$ ). (F) Calcein-AM cell viability assay/Hoechst 33342 staining and H\&E/IHC staining. Blue nuclei comprise both living and dead nuclei, while green sections represent the cytoplasm of living cells. IHC staining indicated the percentage of cells expressing the viability marker Ki67. Scale bars, $100 \mu \mathrm{m}$. (G) The living cell ratios of fresh and warmed tissues. The ratios were found to be not statistically different by Student's t-test $(\mathrm{P}=0.38)$. (H) Cell Counting Kit- 8 assay of in vitro slice cultures before and after cryopreservation. 
I

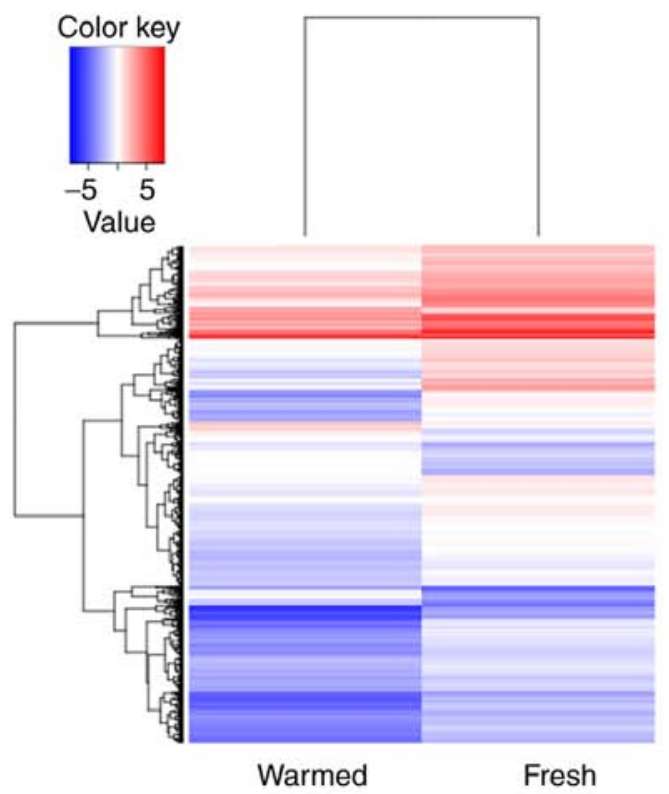

$J$
Enrichment of KEGG

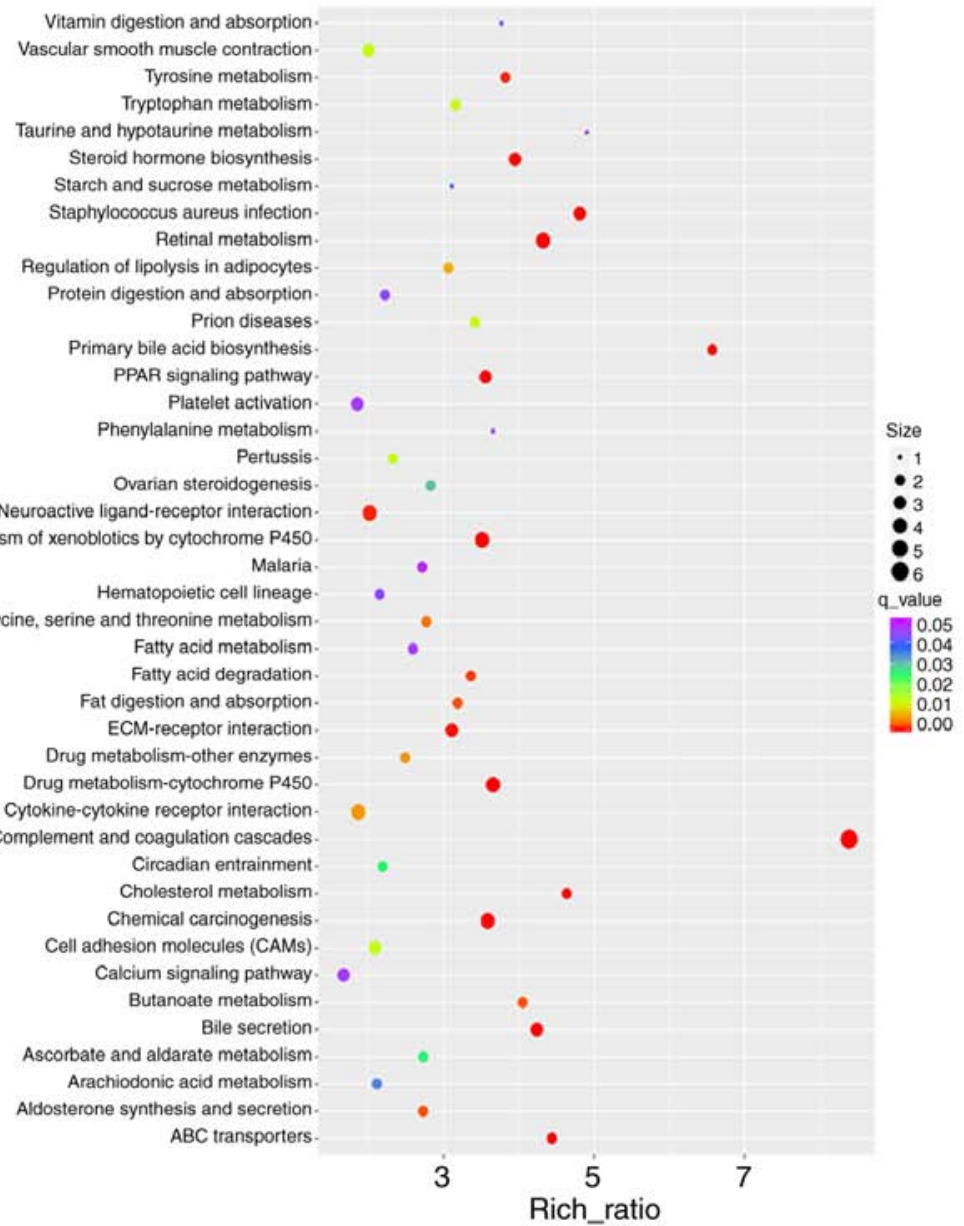

Figure 2. Continued. (I) Heat map of mRNA sequencing. The color change in the heat map is defined as the difference in gene expression between warmed and fresh tissues. Each row represents one gene, and each column represents one type of sample. Compared to the areas of blue color, red color represents increased gene expression. The deeper the red, the more greatly increased the gene expression. The deeper the blue, the lesser the gene expression. (J) KEGG enrichment analysis. In the KEGG diagram, the size of each dot represents the number of differential genes in the corresponding pathway. The different colors of each dot indicate the different degrees of KEGG enrichment. Red represents the highest KEGG enrichment degree. V, vitrification solution; T, warming solution; calcein-AM, acetoxymethyl ester of calcein; H\&E, hematoxylin and eosin; OD, optical density; KEGG, Kyoto Encyclopedia of Genes and Genomes; IHC staining, immunohistochemical staining.

and drug testing were conducted, and repeated three times. The calcein-AM cell viability assay/Hoechst 33342 staining and H\&E/IHC staining indicated that no obvious differences were detectable after $24 \mathrm{~h}$ of slice culturing before drug testing (Fig. 4A, row 1; Fig. 4B, rows 1 and 3; Fig. 4C, P>0.05). However, compared with slices without drug treatment, both fresh and warmed tissue slices cultured for $96 \mathrm{~h}$ showed a marked decrease in cell viability by IHC staining when treated with drugs (Fig. 4A, row 2). Morphological features visible by H\&E staining and Ki67 staining were also changed in both fresh and warmed slices treated with OXA, while they were retained in control slices (Fig. 4B, row 2). Accordingly, a significant decline in Ki67 was detected by IHC staining in slices treated with drugs, while no significant change was found in the control (Fig. 4B, row 4). Furthermore, tissue slices treated for $96 \mathrm{~h}$ with anticancer drugs showed a specific time-dependent reduction in tissue viability by CCK-8 assay (Fig. 4C). Besides, it was found that fresh and warmed tissues both showed evident responses to OXA $(\mathrm{P}<0.05)$, which indicated that the cryopreservation technique had little influence on the tumor biology. Above all,
A
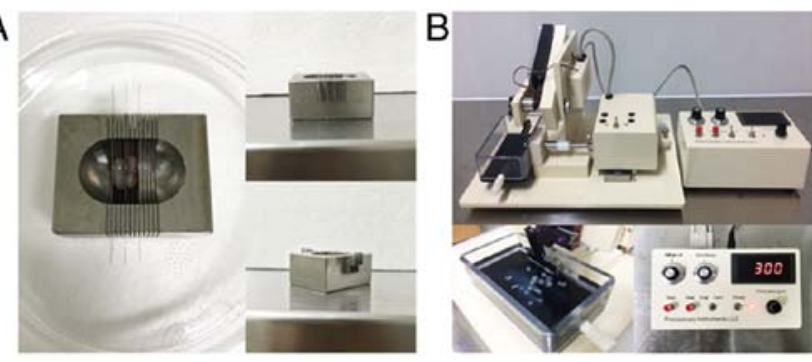

D

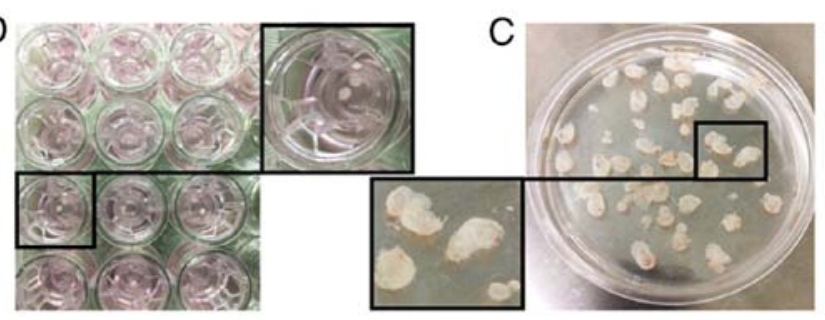

Figure 3. Preparation of precision-cut tissue slices. (A) Metal mold. (B) VF-300 microtome. The sink part was filled with sterile PBS to hold the slice. (C) The precision-cut slices prepared using the microtome. (D) Transwell insert. 
A

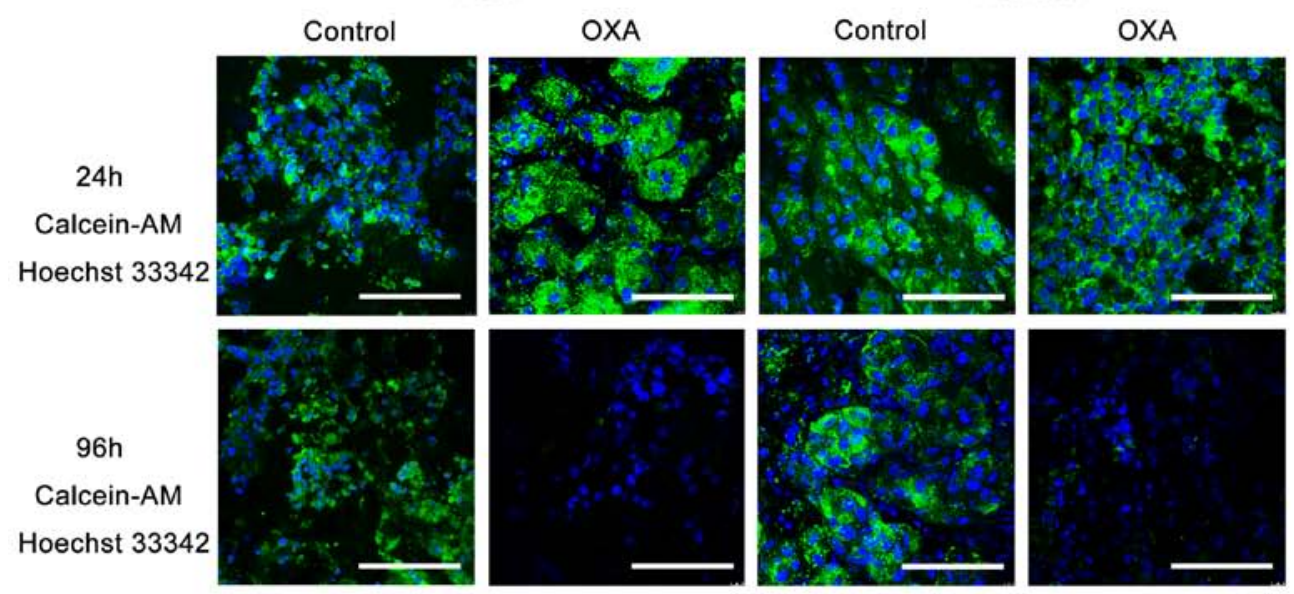

B

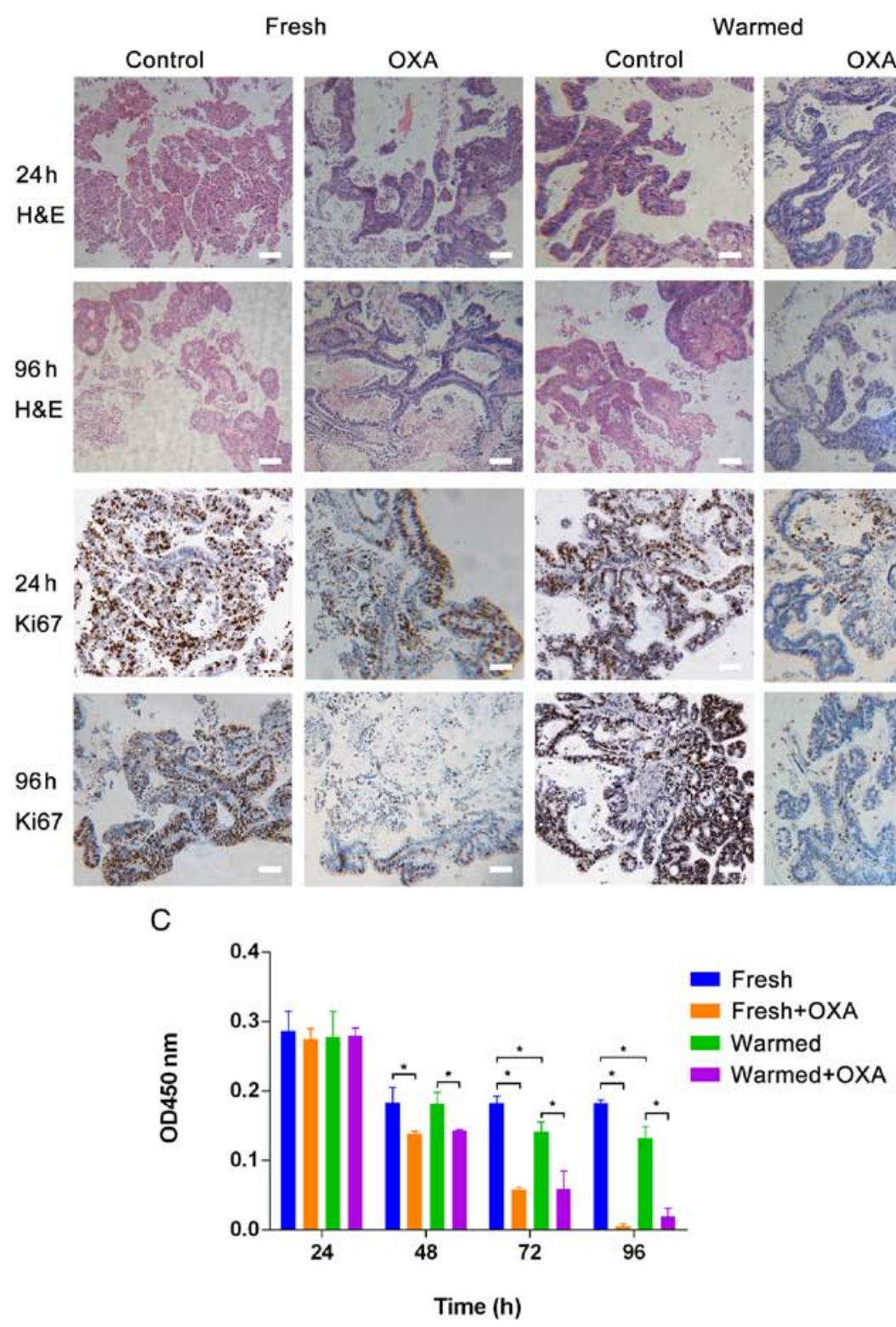

Figure 4. Drug testing in precision-cut slice cultures. (A) Calcein-AM cell viability assay/Hoechst 33342 staining. Scale bars, $100 \mu \mathrm{m}$. (B) H\&E/immunohistochemical staining. Scale bars, $100 \mu \mathrm{m}$. (C) CCK-8 assay of fresh and warmed tissue slice cultures treated with OXA. No obvious difference was detected after $24 \mathrm{~h}$ of slice culturing. After $48 \mathrm{~h}$, the OD values of the fresh group were similar to those in the warmed group, while the OD values of the fresh group were different from those of the fresh + OXA group. Also, the OD values of the warmed group were different from those of the warmed + OXA group. After $72 \mathrm{~h}$, the OD values of the fresh group were different to those of the warmed group. Also, the OD values of the no drug group were different from those of the drug group. After $96 \mathrm{~h}$, the result was the same as at $72 \mathrm{~h} .{ }^{*} \mathrm{P}<0.05$. Calcein-AM, acetoxymethyl ester of calcein; H\&E, hematoxylin and eosin; OXA, oxaliplatin; OD, optical density. 
A
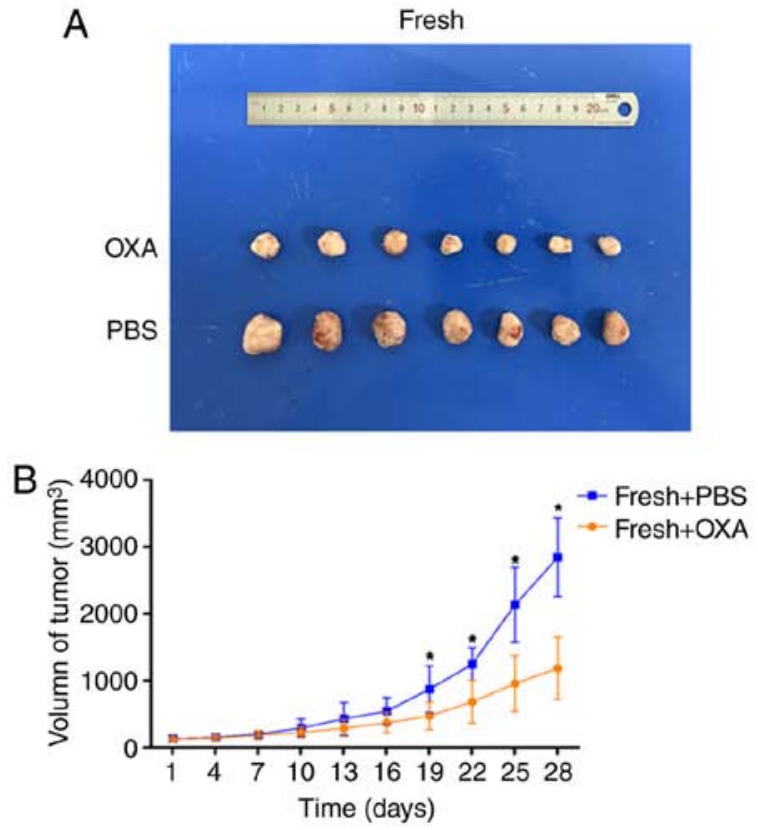

C
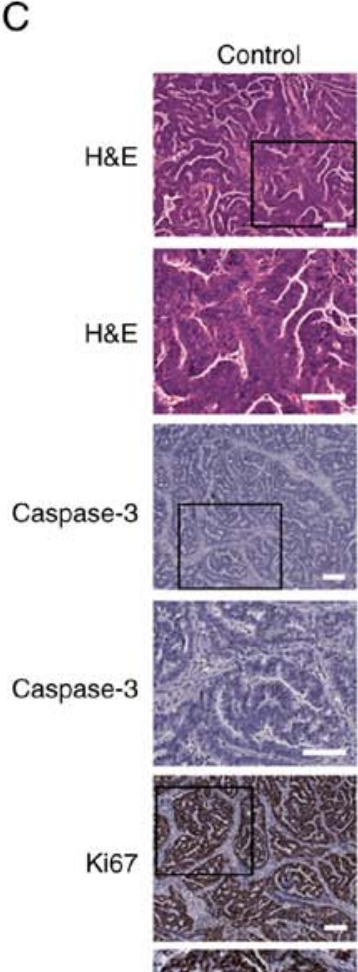

Ki67

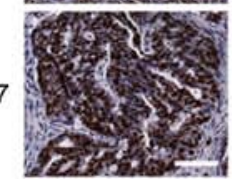

Fresh
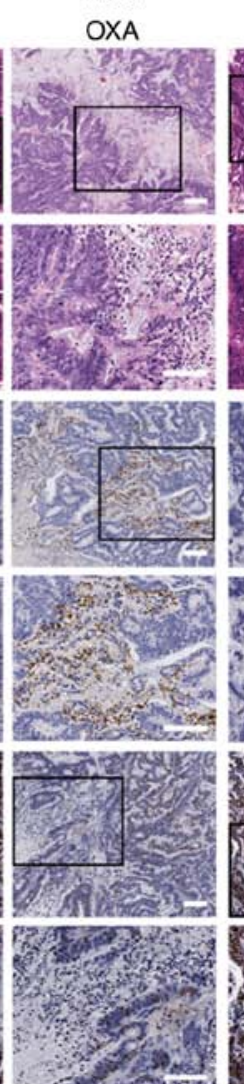

PBS
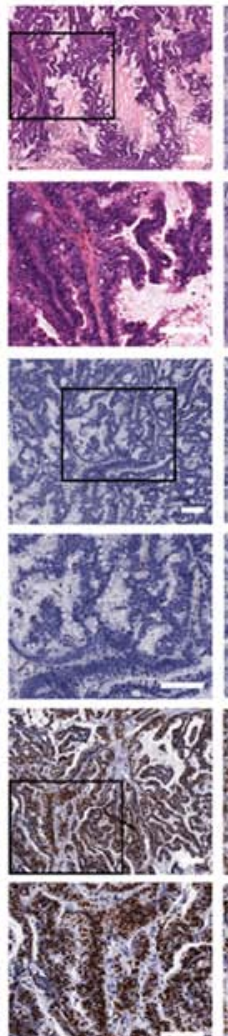
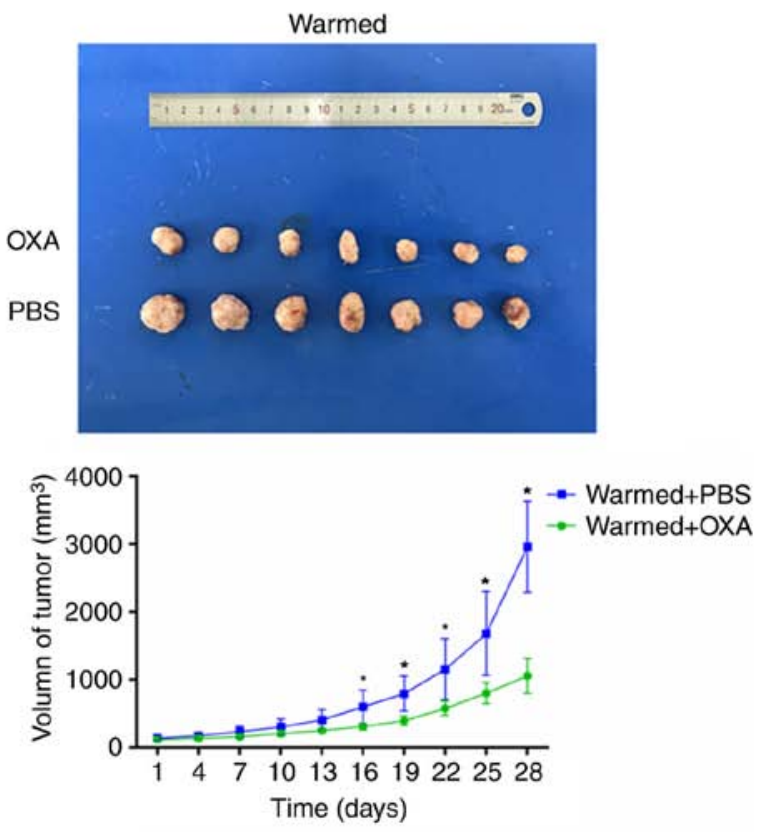

Warmed

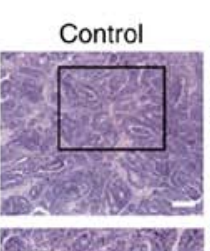

OXA
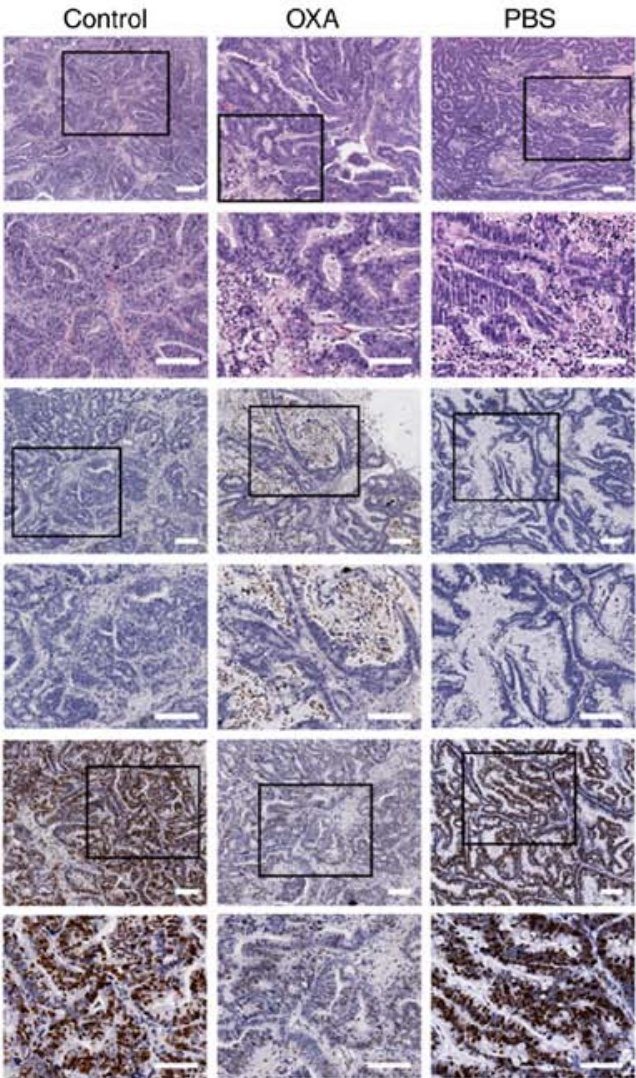

Figure 5. Drug testing in the xenograft model. (A) Gross view of subcutaneous xenograft tumors. (B) Growth curves of subcutaneous xenograft tumor size. The sizes of the tumors in the drug treatment group and control group were statistically different according to the results of the one-way ANOVA. $\mathrm{P}<0.05$ vs. respective Fresh + OXA group or Warmed + OXA group. (C) H\&E and IHC staining of tumor slices from fresh and warmed tissue-derived patient-derived xenografts. Slices were either untreated (control), or treated with OXA or PBS. Scale bars, $100 \mu \mathrm{m}$. IHC staining showed the expression of cleaved Caspase 3 and Ki67. Scale bars, $100 \mu \mathrm{m}$. H\&E, hematoxylin and eosin; IHC staining, immunohistochemical staining; OXA, oxaliplatin.

the precision-cut slices retained the three-dimensional architecture and tumor heterogeneity, and may therefore represent an optimal tool for in vitro drug testing.

Positive drug responses in xenograft models are consistent with those in slice cultures in vitro. In order to examine the concordance of anticancer activity of OXA in vivo and in vitro, PDX models were established. The PDX mice treated with drugs showed a significant difference in tumor size, compared with the mice treated with PBS (Fig. 5A and B; P<0.05). H\&E staining showed an obvious difference in the morphological characteristics of the tumors between mice treated with drugs 
and control mice (Fig. 5C, rows 1 and 2). Accordingly, IHC staining showed increased expression of cleaved caspase-3 and decreased expression of the viability marker Ki-67 in warmed slices treated with OXA, compared to those treated with PBS (Fig. 5C, rows 3-6). Therefore, it was concluded that the drug responses in vivo were in accordance with those of the slice cultures in vitro, indicating the effectiveness of the precision-cut slice model for drug response assessment.

\section{Discussion}

Tumors are intricate and heterogeneous pathological 'organs', which have a close connection with their hosts (11). Various models of cancer in vitro have been applied to the research of cancer biology and drug testing (12-16). However, $\sim 95 \%$ of new anticancer drugs eventually fail in clinical trials (9), because these models fail to maintain the essential features of the heterogeneous architecture of the original tumor. Therefore, innovative models that better capture the key characteristics of heterogeneous tumors, and mimic the interactions between tumor cells and their microenvironment, are required. In the present study, human RLM biopsy tissues were used for the first time, to the best of our knowledge, to establish models in vitro and in vivo to evaluate anticancer drug responses, combining vitrification-based cryopreservation methods with precision-cut slice cultivation. It was specially found that the standardized vitrification method, as an optimized cryopreservation technique, could effectively maintain the biological activity and histological features of the RLM tissues and substantially improve the efficiency of tumor specimen utilization. In addition, with the precision-cut slice culture method in vitro, it was possible to provide three-dimensional architecture and tumor heterogeneity, which may lead to an improvement in the positive response rate of drug testing.

Up to now, only a few studies have reported on the long-term preservation of living tumor tissues, such as human embryos and ovaries (17). Vitrification of biopsy tissues, especially RLM biopsies, has not been reported in the literature to the best of our knowledge. Currently, most PDX models have been established from fresh surgical resections (18-20). In the present study, PDX models were established successfully using both fresh and warmed RLM biopsy tissues, indicating that cryopreservation of both fresh and warmed biopsy tissues is feasible and efficient. By conducting several CCK- 8 assays in the earlier stages of the research, it was found that no difference was induced by different lengths of preservation time in liquid nitrogen. However, the whole cryopreservation and warming procedure should be conducted strictly in accordance with the time scheme used in the present study. Additionally, the fresh biopsy should be transported to the laboratory within $2 \mathrm{~h}$, which is critical to the activity of the living tissue. Since the amount of biopsy tissues was limited in the present study, it was determined to first cryopreserve the biopsy tissues and then establish the xenografts to generate enough tissues for further experiments. The results of the present study showed that the novel vitrification-based cryopreservation method is capable of retaining the proliferative capacity and morphological characteristics of the original tumor. No obvious difference was detected in the biological characteristics of the tissues before and after cryopreservation. According to the heat map generated, it was clear that the amount of differential gene expression was limited. Furthermore, the KEGG enrichment analysis revealed that the majority of the differential genes were associated with metabolism; it is hypothesized that the large changes in temperature during the cryopreservation and warming procedures may be the main reason for this. Compared with conventional specimen preservation methods, the novel vitrification-based cryopreservation method is likely to efficiently improve viability after warming. Notably, this method avoided ice crystallization, by which the cell viability may be reduced (21). Furthermore, the required time and basic cost of this cryopreservation method were lower than those of conventional cryopreservation methods (22).

Precision-cut slices have been applied to set up micro-tissue culture systems in vitro, which can be used to perform preclinical and clinical studies on novel therapies or for basic research $(18,23)$. Slices of $300 \mu \mathrm{M}$ were thought to be the most suitable thickness for RLM after conducting several slicing experiments with different thicknesses. The most prominent merit of precision-cut slicing was that the full heterogenous architecture of the tissues was retained, and slice-based drug testing avoided the potential damaging effects of the process of tumor cell isolation from the biological environment $(24,25)$. In the present study, the tissue slices processed by microtome all showed evident responses to anticancer drugs. Furthermore, drug responses in the PDXs were consistent with those in the slice cultures in vitro. Compared with the PDX model, tissue slice cultivation represented a more convenient and practical model in light of the shorter culture cycle and simpler operation. Certainly, the insert support was helpful for maintaining slice viability, and enabled the implementation of a steady platform and provided an ample supply of oxygen and nutrition, compared with floating alone $(18,26,27)$. On the whole, precision-cut slice cultivation retained the heterogeneous architecture of the original tumor and thus might provide a basis for in-depth study of pharmacokinetic mechanisms in pharmacology and gene pathways in tumor biology.

The present study standardized a workflow for the cryopreservation and warming of RLM biopsy tissues, combined with precision-cut slice cultivation to assess anticancer drug responses. The present study demonstrated that biopsy tissues from RLM could be effectively cryopreserved, and that the tumor biological characteristics were well retained. In addition, precision-cut slices derived from the warmed tissues provided an efficient tool to assess anticancer drug responses in vitro. Above all, the cryopreserved biopsy tissues combined with precision slice culture represented an optimal method of investigating personalized therapeutics. Further studies to evaluate this model for novel preclinical and clinical drug trials are warranted.

\section{Acknowledgements}

The authors would like to thank Dr Tao Wang for technical assistance.

\section{Funding}

This study was funded by the National Natural Science Foundation of China (grant no. 81472845). 


\section{Availability of data and materials}

The datasets used and/or analyzed during the current study are available from the corresponding author on reasonable request.

\section{Authors' contributions}

YZ conceived and designed the study, conducted the experiments and drafted the article. WJH performed the H\&E/IHC staining of tissue and was involved in drafting the manuscript. QRY, HDZ and XJZ performed the statistical analysis. XZ and $M Z$ made substantial contributions to the conception and design. ZYW, WJL, HSJ, XBZ, YPS, HH and HXY were involved in drafting the manuscript and revising it critically for important intellectual content. ZHL made substantial contributions to the design and agreed to be accountable for all aspects of the work in ensuring that questions related to the accuracy or integrity of any part of the work are appropriately investigated and resolved. BZ was involved in drafting and revising the manuscript, made substantial contributions to the design, and approved the final version to be submitted. All authors read and approved the final manuscript.

\section{Ethics approval and consent to participate}

The investigation was approved by ethics committee of Renji Hospital and all patients provided written informed consent. All the experimental procedures performed on the animals were approved by the Shanghai Medical Experimental Animal Care Commission. Informed consent was obtained from all individual participants included in the study.

\section{Patient consent for publication}

Not applicable.

\section{Competing interests}

The authors declare that they have no competing interests.

\section{References}

1. Ozyurt H, Ozden AS, Ozgen Z, Gemici C and Yaprak G: Pre- and post-surgery treatments in rectal cancer: A long-term single-centre experience. Curr Oncol 24: e24-e34, 2017.

2. Kim KH, Shin SJ, Cho MS, Ahn JB, Jung M, Kim TI, Park YS, Kim H, Kim NK and Koom WS: A phase II study of preoperative mFOLFOX6 with short-course radiotherapy in patients with locally advanced rectal cancer and liver-only metastasis. Radiother Oncol 118: 369-374, 2016.

3. De Greef K, Rolfo C, Russo A, Chapelle T, Bronte G, Passiglia F, Coelho A, Papadimitriou K and Peeters M: Multisciplinary management of patients with liver metastasis from colorectal cancer. World J Gastroenterol 22: 7215-7225, 2016.

4. Loree JM and Kopetz S: Recent developments in the treatment of metastatic colorectal cancer. Ther Adv Med Oncol 9: 551-564, 2017.

5. Burdall SE, Hanby AM, Lansdown MR and Speirs V: Breast cancer cell lines: Friend or foe? Breast Cancer Res 5: 89-95, 2003.

6. Naipal KA, Verkaik NS, Sanchez H, van Deurzen $\mathrm{CH}$, den Bakker MA, Hoeijmakers JH, Kanaar R, Vreeswijk MP, Jager A and van Gent DC: Tumor slice culture system to assess drug response of primary breast cancer. BMC Cancer 16: 78, 2016 .
7. Usui T, Sakurai M, Enjoji S, Kawasaki H, Umata K, Ohama T, Fujiwara N, Yabe R, Tsuji S, Yamawaki H, et al: Establishment of a novel model for anticancer drug resistance in three-dimensional primary culture of tumor microenvironment. Stem Cells Int 2016: 7053872, 2016.

8. van der Kuip H, Murdter TE, Sonnenberg M, McClellan M, Gutzeit S, Gerteis A, Simon W, Fritz P and Aulitzky WE: Short term culture of breast cancer tissues to study the activity of the anticancer drug taxol in an intact tumor environment. BMC Cancer 6: 86, 2006.

9. Hickman JA, Graeser R, de Hoogt R, Vidic S, Brito C, Gutekunst $\mathrm{M}$ and van der Kuip H; IMI PREDECT Consortium: Three-dimensional models of cancer for pharmacology and cancer cell biology: Capturing tumor complexity in vitro/ex vivo. Biotechnol J 9: 1115-1128, 2014.

10. Holliday DL, Moss MA, Pollock S, Lane S, Shaaban AM, Millican-Slater R, Nash C, Hanby AM and Speirs V: The practicalities of using tissue slices as preclinical organotypic breast cancer models. J Clin Pathol 66: 253-255, 2013.

11. Egeblad M, Nakasone ES and Werb Z: Tumors as organs: Complex tissues that interface with the entire organism. Dev Cell 18: 884-901, 2010.

12. Barretina J,CaponigroG, Stransky N, Venkatesan K, Margolin AA, Kim S, Wilson CJ, Lehár J, Kryukov GV, Sonkin D, et al: The cancer cell line encyclopedia enables predictive modelling of anticancer drug sensitivity. Nature 483: 603-607, 2012.

13. Fiebig HH, Maier A and Burger AM: Clonogenic assay with established human tumour xenografts: Correlation of in vitro to in vivo activity as a basis for anticancer drug discovery. Eur J Cancer 40: 802-820, 2004.

14. Lee JM, Mhawech-Fauceglia P, Lee N, Parsanian LC, Lin YG, Gayther SA and Lawrenson K: A three-dimensional microenvironment alters protein expression and chemosensitivity of epithelial ovarian cancer cells in vitro. Lab Invest 93: 528-542, 2013.

15. Lin RZ and Chang HY: Recent advances in three-dimensional multicellular spheroid culture for biomedical research. Biotechnol J 3: 1172-1184, 2008.

16. Chen $\mathrm{HC}$ and $\mathrm{Hu} \mathrm{YC}$ : Bioreactors for tissue engineering. Biotechnol Lett 28: 1415-1423, 2006.

17. Zhou XH, Zhang D, Shi J and Wu YJ: Comparison of vitrification and conventional slow freezing for cryopreservation of ovarian tissue with respect to the number of intact primordial follicles: A meta-analysis. Medicine (Baltimore) 95: e4095, 2016.

18. Chadwick EJ, Yang DP, Filbin MG, Mazzola E, Sun Y, Behar O, Pazyra-Murphy MF, Goumnerova L, Ligon KL, Stiles CD and Segal RA: A brain tumor/organotypic slice co-culture system for studying tumor microenvironment and targeted drug therapies. J Vis Exp: e53304, 2015.

19. Unger F, Bentz S, Kruger J, Rosenbrock C, Schaller J, Pursche K, Sprüssel A, Juhl H and David KA: Precision cut cancer tissue slices in anti-cancer drug testing. J Mol Pathophysiol 4: 108-121, 2015.

20. Bruna A, Rueda OM, Greenwood W, Batra AS, Callari M, Batra RN, Pogrebniak K, Sandoval J, Cassidy JW, TufegdzicVidakovic A, et al: A biobank of breast cancer explants with preserved intra-tumor heterogeneity to screen anticancer compounds. Cell 167: 260-274 e22, 2016.

21. Elder E, Chen Z, Ensley A, Nerem R, Brockbank K and Song Y: Enhanced tissue strength in cryopreserved, collagen-based blood vessel constructs. Transplant Proc 37: 4625-4629, 2005.

22. Zeng M, Yang QR, Fu GB, Zhang Y, Zhou X, Huang WJ, Zhang HD, Li WJ, Wang ZY, Yan HX and Zhai B: Maintaining viability and characteristics of cholangiocarcinoma tissue by vitrification-based cryopreservation. Cryobiology 78: 41-46, 2017.

23. Bourke S, Mason HS, Borok Z, Kim KJ, Crandall ED and Kemp PJ: Development of a lung slice preparation for recording ion channel activity in alveolar epithelial type I cells. Respir Res 6: 40, 2005.

24. Pietras K and Ostman A: Hallmarks of cancer: Interactions with the tumor stroma. Exp Cell Res 316: 1324-1331, 2010.

25. Hanahan D and Weinberg RA: Hallmarks of cancer: The next generation. Cell 144: 646-674, 2011.

26. Davies EJ, Dong M, Gutekunst M, Närhi K, van Zoggel HJ, Blom S, Nagaraj A, Metsalu T, Oswald E, Erkens-Schulze S, et al: Capturing complex tumour biology in vitro: Histological and molecular characterisation of precision cut slices. Sci Rep 5: 17187, 2014.

27. Lima D, Silva T, Morais GB, Aquino-Cortez A, Evangelista J, Xavier Júnior F, Viana DA and Silva L: Different associations of cryoprotectants for testicular tissue of prepubertal cats submitted to vitrification. Reprod Domest Anim 52 (Suppl 2): S235-S241, 2017.

(i) (2) This work is licensed under a Creative Commons Attribution-NonCommercial-NoDerivatives 4.0 International (CC BY-NC-ND 4.0) License. 